\title{
Autologous Deep IL-15 Primed T-cells TRQ15-01
}

National Cancer Institute

\section{Source}

National Cancer Institute. Autologous Deep IL-15 Primed T-cells TRQ15-01. NCI

Thesaurus. Code C158091.

A preparation of genetically modified, multi-antigen-directed autologous T-lymphocytes, that have particles, consisting of multiple chemically crosslinked human cytokine interleukin-15 (IL-15)/IL-15 receptor alpha (IL-15Ra)/Fc heterodimers, attached to their surface, with potential immunostimulating and antineoplastic activities. TRQ15-01 is made from monocyte-derived dendritic cells (moDCs) that are pulsed with peptides from multiple tumor-associated antigens (TAAs) to expand cytotoxic T-lymphocytes (CT Ls) that are subsequently loaded with IL-15 particles. Upon administration of the autologous deep IL-15 primed T-cells, the IL-15/IL-15Ra fusion proteins are slowly released in vivo from the T-cells in a controlled manner and induce autocrine cytokine stimulation of the administered T-cells, thereby increasing T-cell division of the administered T-cells. The expanded T-cells target, bind to and kill tumor cells. This increases tumor cell growth inhibition by T-cells. IL-15 is a pro-survival, inflammatory cytokine and causes sustained T-cell expansion and enhanced anti-tumor activity. Compared to systemically delivered IL-15, IL-15 attached to the T-cells greatly increases target CD8 T-cell concentrations in the tumor, without significant systemic effects. 